\title{
High-Sensitivity and High-Resolution SPECT/CT Systems Provide Substantial Dose Reduction Without Compromising Quantitative Precision for Assessment of Myocardial Perfusion and Function
}

\author{
Richard J. Palyo ${ }^{1}$, Albert J. Sinusas ${ }^{2,3}$, and Yi-Hwa Liu ${ }^{1,2,4,5}$ \\ ${ }^{1}$ Nuclear Cardiology, Heart and Vascular Center, Yale-New Haven Hospital, New Haven, Connecticut; ${ }^{2}$ Section of Cardiovascular \\ Medicine, Department of Internal Medicine, Yale University School of Medicine, New Haven, Connecticut; ${ }^{3}$ Department of Radiology \\ and Biomedical Imaging, Yale University School of Medicine, New Haven, Connecticut; ${ }^{4}$ Department of Biomedical Imaging and \\ Radiological Sciences, National Yang-Ming University, Taipei, Taiwan; and ${ }^{5}$ Department of Biomedical Engineering, Chung Yuan \\ Christian University, Taoyuan, Taiwan
}

There is increasing concern about radiation exposure from myocardial perfusion SPECT (MPS). y-cameras with solid-state cadmiumzinc-telluride (CZT) detectors have better count sensitivity and spatial resolution than conventional sodium iodine detectors, allowing for significant reductions in radiotracer dose or acquisition time. This study aimed to demonstrate the capability of a hybrid CZT SPECT/ 64-slice CT system for dose reduction and to determine the maximal reduction possible without compromising image quality or the quantification precision of clinical MPS. Methods: The imaging data of patients with normal myocardial perfusion and 30 patients with midsized to large perfusion defects who had undergone stress ${ }^{99 m} \mathrm{Tc}-$ tetrofosmin MPS were postprocessed. Low-dose (361 $\pm 60 \mathrm{MBq})$

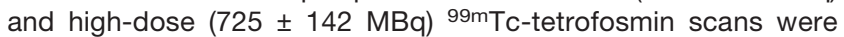
included, with 6-min and 4-min scanning times, respectively. List-mode SPECT data were reconstructed with CT-based attenuation correction and with full as well as $50 \%$ and $75 \%$ reductions in acquisition time to simulate the corresponding relative dose reductions. The reconstructed SPECT images were analyzed to calculate global MPS defect size and regional defect size for 3 coronary artery territories-left anterior descending, left circumflex, and right-as well as left ventricular (LV) volume and ejection fraction. Results: For patients with normal MPS results, there were no differences in defect size, LV volume, or ejection fraction, regardless of whether $50 \%$ or $75 \%$ reduction was used. For patients with abnormal MPS results, at a $50 \%$ reduction there was a significant difference in global defect size but not in regional defect size in the left anterior descending, left circumflex, and right coronary artery territories, whereas at a $75 \%$ reduction the difference was statistically significant in all territories, including the difference in global defect size. Nonetheless, differences in the defect size were minimal. The LV end-diastolic and end-systolic volumes and LV ejection fraction were not significantly different, regardless of whether $50 \%$ or $75 \%$ dose reduction was used. Conclusion: Ultra-low-dose ( $<190 \mathrm{MBq})$ MPS even with short imaging times ( $<6 \mathrm{~min}$ ) is feasible using a hybrid CZT SPECT/CT camera without compromising image quality or significantly altering quantification of myocardial perfusion or LV function. We demonstrated

Received Aug. 3, 2015; revision accepted Jan. 5, 2016.

For correspondence or reprints contact: Yi-Hwa Liu, Section of Cardiovascular Medicine, Department of Internal Medicine, Yale University School of Medicine, 333 Cedar St., P.O. Box 208017, New Haven, CT 06520. E-mail: yi-hwa.liu@yale.edu

Published online Feb. 4, 2016.

COPYRIGHT (C) 2016 by the Society of Nuclear Medicine and Molecular Imaging, Inc. that an additional 50\% reduction in the current low-dose recommendations from the American Society of Nuclear Cardiology guidelines for ${ }^{99 \mathrm{~m} T c-l a b e l e d ~ M P S}$ is highly feasible while retaining short imaging protocols.

Key Words: dose reduction; SPECT quantification; myocardial perfusion; left ventricular function

J Nucl Med 2016; 57:893-899

DOI: 10.2967/jnumed.115.164632

O

PECT with ${ }^{99 m}$ Tc-labeled radiotracers has been widely used for the detection of abnormalities of myocardial perfusion and left ventricular (LV) function (1-9). Hybrid SPECT/CT systems equipped with a multislice $\mathrm{CT}$ component allowing for precise attenuation correction (AC) (10-12) have been used routinely in nuclear cardiac imaging for more than a decade. Although this imaging modality is well recognized to provide invaluable diagnostic information about coronary artery diseases, radiation exposure to patients has become a growing concern (13). Reduction of radiation exposure is currently a major focus in the realm of nuclear cardiology.

Previous studies (14-20) have shown that the dose of radiotracer injected into patients can be reduced and acquisition time can be shortened using new imaging technologies with highly efficient $\gamma$-detection and dedicated small-field-of-view image reconstruction $(9,21)$. More specifically, extraordinarily sensitive SPECT cameras with solid-state cadmium-zinc-telluride (CZT) detectors have better count sensitivity and spatial resolution than those with conventional sodium iodine detectors. Also, these $\gamma$-cameras are capable of acquiring images in list mode $(9,21,22)$ to facilitate data selection for image reconstruction. Software for these imaging modalities allows for a variety of postacquisition reprocessing, including selection of a portion of the entire scanned dataset and reconstruction of the image data from such subselections. Although promising observations have been made for a similar type of system $(15,20,23)$, detailed quantitative SPECT analysis with AC has not been extensively explored.

The aims of this clinical study were, first, to demonstrate the capability of a new cardiac SPECT/CT system equipped with CZT $\gamma$-detectors to reduce the injected dose of radiotracer and the exposure of patients to radiation and, second, to determine a 
potential upper boundary on dose reduction that does not compromise quantitative image analyses in myocardial perfusion SPECT (MPS).

\section{MATERIALS AND METHODS}

\section{Patient Selection}

Ten patients ( 3 male and 7 female) with low-risk and normal MPS results and 30 patients (15 male and 15 female) with abnormal MPS results were randomly selected from the Yale-New Haven Hospital nuclear cardiac imaging database to have SPECT postprocessing applied to their clinical MPS studies. The body mass index ranged from 17.8 to $34.4 \mathrm{~kg} / \mathrm{m}^{2}$ for the normal group and 19.0 to $36.6 \mathrm{~kg} / \mathrm{m}^{2}$ for the abnormal group. The inclusion criteria were that the images of each clinically indicated study had been acquired using a hybrid SPECT/ CT camera (Discovery 570c; GE Healthcare) with the capability of CT-based AC and that the studies showed either normal myocardial perfusion $(0 \%$ of the left ventricle) or a moderate $(5 \%-10 \%$ of the left ventricle) to large ( $>10 \%$ of the left ventricle) myocardial perfusion defect as defined using the Yale University circumferential quantitative profile analysis software, which calculates an integrated myocardial perfusion defect size (\%LV). We included in our analysis both normal and abnormal MPS studies to investigate the potential that dose reduction will generate false-positive scans in low-risk patients or changes in quantitative integrated perfusion defect size in patients with perfusion abnormalities. Only stress acquisitions were included for this SPECT postprocessing because they were more likely than the corresponding rest acquisitions to demonstrate heterogeneity in myocardial radiotracer uptake on SPECT images. The Institutional Review Board approved this retrospective study, and the requirement to obtain informed consent was waived.

\section{Patient Preparation and Stress Protocol}

Patients were prohibited from ingesting food, beverages, or medicine for at least $4 \mathrm{~h}$ before the stress tests. Exercise stress testing was performed on a treadmill, with $85 \%$ of target heart rate being a minimum requirement before administration of the ${ }^{99 \mathrm{~m}} \mathrm{Tc}$-tetrofosmin (Myoview; GE Healthcare) radiotracer injection. For patients who could not exercise, pharmacologic stress ${ }^{99 \mathrm{~m} T c-t e t r o f o s m i n ~ i m a g i n g ~ w a s ~ p e r f o r m e d ~}$ after regadenoson (Lexiscan; Astellas US LLC) stress. All stress testing was performed with serial 12-lead electrocardiogram monitoring and serial blood pressure monitoring throughout the stress protocol and recovery time. Among the 10 normal-perfusion patients, 5 were injected with a low dose $(337 \pm 15 \mathrm{MBq})$ and the other 5 with a high dose $(712 \pm 39 \mathrm{MBq})$ of ${ }^{99 \mathrm{~m}} \mathrm{Tc}$-tetrofosmin. Among the 30 abnormalperfusion patients, 22 were injected with a low dose $(384 \pm 58 \mathrm{MBq})$ and the other 8 with a high dose $(737 \pm 137 \mathrm{MBq})$ of ${ }^{99 \mathrm{~m}} \mathrm{Tc}$-tetrofosmin.

\section{Image Acquisition and Processing}

All images were acquired in list mode using the hybrid SPECT/CT camera at the Yale-New Haven Hospital laboratory. This camera contains an array of 19 solid-state CZT $\gamma$-detectors, each with a 5-mm pinhole collimator for SPECT imaging, and is also equipped with a 64-slice CT component for SPECT AC. The CZT detectors are aligned across the gantry to acquire SPECT images at 19 separate angles converging to a spheric field of view (19 $\mathrm{cm}$ in diameter), allowing for full coverage of the heart with one simultaneous acquisition. The peak of the energy

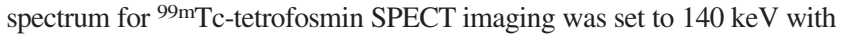
$\mathrm{a} \pm 10 \%$ window, and the matrix was $32 \times 32$ pixels (pixel size, $2.5 \mathrm{~mm}$ ) for acquisition and $70 \times 70$ pixels (pixel size, $4 \mathrm{~mm}$ ) for reconstruction. The acquired SPECT list-mode data were rebinned into various acquisition times to simulate different levels of radiation exposure, which were presumably linearly correlated with the injected radiotracer dose used. For CT imaging, a voltage of $120 \mathrm{kVp}$, a current of $50 \mathrm{~mA}$, a rotation time of $0.4 \mathrm{~s}$, detector coverage of $40 \mathrm{~mm}$, a slice thickness of $2.5 \mathrm{~mm}$, and a pitch of 0.984:1 (39.37-mm coverage per rotation) were used.

Patients were positioned in the camera gantry such that the heart was centered in the field of view of the CZT SPECT detectors. Images of the patients injected with low doses were acquired for 6 min, whereas those with the high doses were acquired for $4 \mathrm{~min}$. To assess LV function, SPECT acquisitions were electrocardiogram-gated into 16 bins with a $50 \%$ beat rejection window. The acquired images were reconstructed using maximum-likelihood expectation maximization in list mode with the full acquisition time (100\%), as well as with $50 \%$ and $75 \%$ (i.e., $50 \%$ and $25 \%$ of full acquisition time, respectively) reductions from the original imaging times to simulate $50 \%$ and $75 \%$ reductions of injected dose. Reconstructed SPECT images were analyzed using the Yale SPECT quantification software (24) to calculate global myocardial perfusion defect sizes, LV end-diastolic volume (EDV), end-systolic volume (ESV), and ejection fraction (EF). Moreover, regional defect sizes were also calculated for the territories of the left anterior descending, left circumflex, and right coronary arteries as defined by the 17-segment model (8).

\section{Statistical Analysis}

Linear regression analysis and Bland-Altman (25) analysis of agreement were used to assess correlation and estimation error between the 2 measures. ANOVA was performed for overall comparison among all groups $(0 \%, 50 \%$, and $75 \%$ dose reductions) for each measure. Paired $t$ testing with Bonferroni adjustment was used to evaluate differences between 2 individual groups paired. A difference in this paired comparison was considered statistically significant if the $P$ value was less than 0.016 with the Bonferroni adjustment.

\section{RESULTS}

In the context of this report, the terms dose reduction and acquisition time reduction are interchangeable since the reduction in SPECT acquisition time $(0 \%, 50 \%$, and $75 \%)$ is presumed to have a linear relationship with that in injected dose.

\section{Qualitative and Quantitative Comparisons of SPECT With and Without Dose Reduction}

Illustrated in Figures 1 and 2 are SPECT images and the corresponding circumferential count profiles from 2 representative patient studies: one with normal (Fig. 1) and the other with abnormal (Fig. 2) MPS results. As shown, the SPECT images with 50\% and $75 \%$ dose reduction are visually similar to those without dose reduction. Compared with the original $100 \%$ acquisition time, the circumferential count profiles were almost superimposable when images were reconstructed with the acquisition time reduced by $50 \%$ and $75 \%$. These observations were true in both normal (Fig. 1) and abnormal (Fig. 2) cases. As seen in Figure 1, all circumferential count profiles are above the lower-limit-of-normal count profiles, regardless of whether $50 \%$ or $75 \%$ dose reduction was used. The global defect sizes for low-risk subjects were calculated to be $0 \%$ (i.e., no false-positives), and SPECT-quantified EDV, ESV, and LVEF were similar for the $0 \%, 50 \%$, and $75 \%$ dose reductions (EDV of 58, 58, and $57 \mathrm{~mL}$; ESV of 22, 22, and $22 \mathrm{~mL}$; and LVEF of $63 \%, 61 \%$, and $62 \%$ for the $0 \%, 50 \%$, and $75 \%$ time reductions, respectively). Shown in Figure 2 are SPECT images and the corresponding circumferential count profiles for the abnormal study reconstructed without dose reduction and with $50 \%$ and $75 \%$ dose reduction. The count profiles with dose reduction were similar to those without dose reduction. Although there was a minor difference among the count profiles in the midventricular area (Fig. 2), the SPECT-quantified defect sizes were not markedly different. More specifically, SPECT-quantified global defect size for this case was 


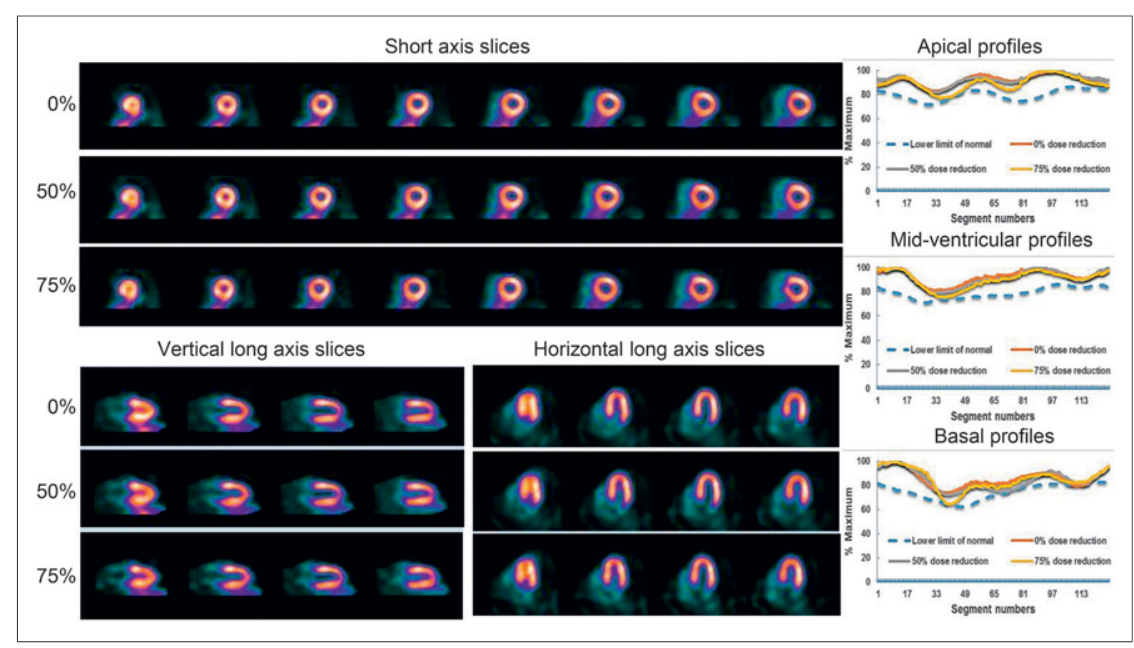

FIGURE 1. Illustrations of SPECT slices and 2-dimensional circumferential count profiles of patients without myocardial perfusion defects, reconstructed and quantified with $0 \%, 50 \%$, and $75 \%$ dose reductions. Two-dimensional count profiles were generated from radial segments of short-axis slices, starting approximately at the 1-o'clock position and continuing counterclockwise.

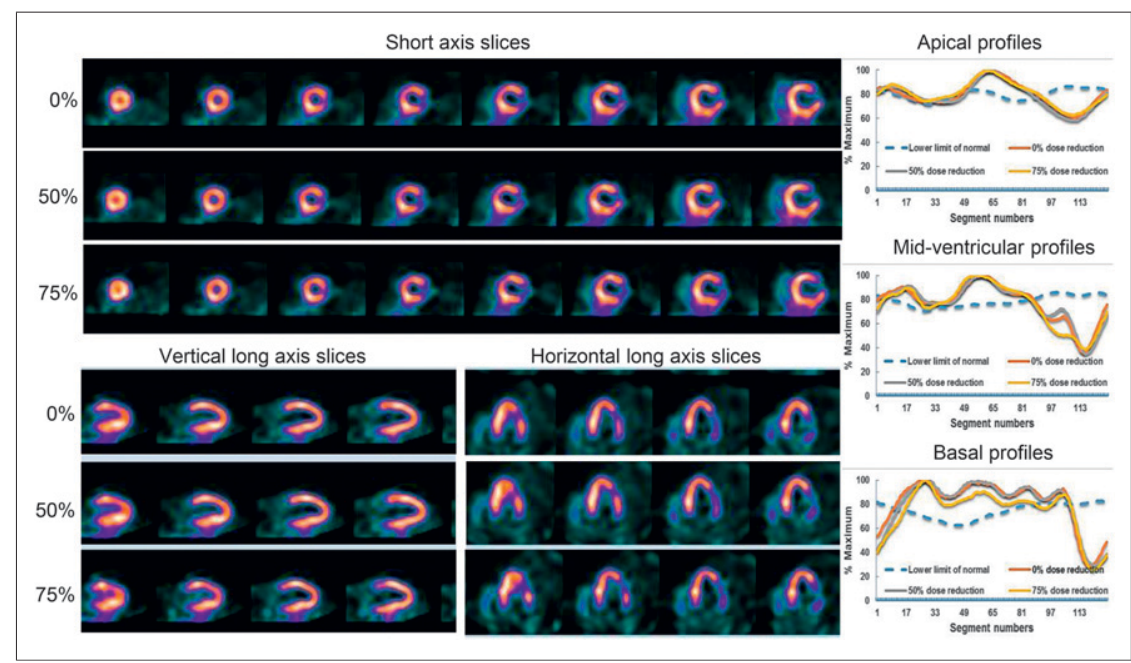

FIGURE 2. Illustrations of SPECT slices and 2-dimensional circumferential count profiles of patients with myocardial perfusion defects, reconstructed and quantified with $0 \%, 50 \%$, and $75 \%$ dose reductions. Two-dimensional count profiles were generated from radial segments of short-axis slices, starting approximately at the 1 -o'clock position and continuing counterclockwise.

$15 \%$ of the left ventricle (large-sized) without dose reduction and remained large - at $16 \%$ and $18 \%$ - with $50 \%$ and $75 \%$ dose reductions, respectively. SPECT-quantified EDV, ESV, and LVEF for the $0 \%, 50 \%$, and $75 \%$ dose reductions for the patient in Figure 2 were also similar (EDV of 98, 99, and $97 \mathrm{~mL}$; ESV of 56, 56, and $55 \mathrm{~mL}$; and LVEF of $56 \%, 56 \%$, and $55 \%$ for the $0 \%, 50 \%$, and $75 \%$ time reductions, respectively).

\section{Effect of Dose Reduction on \\ Myocardial Perfusion Defect Size}

Comparisons of SPECT-quantified global defect sizes among the $0 \%, 50 \%$, and $75 \%$ dose reductions for patients with normal and abnormal MPS results are shown in Tables 1 and 2, respectively. For normal cases, the global defect size remained $0 \%$ as seen in Table 1, regardless of whether $0 \%, 50 \%$, or $75 \%$ dose reduction was used, indicating that the dose reductions did not create a falsepositive defect in these normal-perfusion studies. For patients with myocardial perfusion defects, there was a significant difference in global defect sizes between the $50 \%$ and $75 \%$ dose reductions and the $0 \%$ dose reduction $(P<0.016$, paired $t$ test; $n=$ 30). Nevertheless, as seen in Table 2, the differences were minimal $(<3 \% \mathrm{LV})$ and the mean defect sizes remained within the same defect category. As such, these small differences in defect size without a change in defect-size category might not be clinically significant.

Comparisons of SPECT-quantified global defect sizes among the original images $(0 \%$ dose reduction) and the $50 \%$ and $75 \%$ dose reduction images, reconstructed without and with AC, are shown in Figures 3 and 4, respectively. As seen, the linear regression and Bland-Altman plots resulting from SPECT with and without $\mathrm{AC}$ were similar. Figure 5 and Supplemental Figure 1 show the same analyses as those in Figure 4 except for the individual left anterior descending, left circumflex, and right coronary artery territories. Overall, the correlations were excellent and there were no statistically significant differences ( $n=30$, ANOVA) in the global or regional myocardial perfusion defect sizes among the 3 groups $(0 \%, 50 \%$, and $75 \%$ dose reductions). However, in the paired $t$ analysis, there were statistically

TABLE 1

Defect Size in Patients with Normal Myocardial Perfusion

\begin{tabular}{lcccc}
\hline Dose reduction & Global defect size $(\% \mathrm{LV})$ & EDV $(\mathrm{mL})$ & ESV $(\mathrm{mL})$ & $19 \pm 6$ \\
\hline $0 \%$ & $0 \pm 0$ & $62 \pm 12$ & $19 \pm 6$ & $71 \pm 5$ \\
$50 \%$ & $0 \pm 0$ & $63 \pm 13$ & $19 \pm 6$ & $70 \pm 4$ \\
$75 \%$ & $0 \pm 0$ & $63 \pm 11$ & $70 \pm 5$
\end{tabular}

Data are mean \pm SD. $P=$ not statistically significant with paired $t$ test with Bonferroni adjustment ( $50 \%$ vs. $0 \%$ and $75 \%$ vs. $0 \%) ; n=10$. 
TABLE 2

Defect Size in Patients with Myocardial Perfusion Defects

\begin{tabular}{lcccc}
\hline Dose reduction & Global defect size $(\% \mathrm{LV})$ & EDV $(\mathrm{mL})$ & ESV $(\mathrm{mL})$ & EF $(\%)$ \\
\hline $0 \%$ & $16 \pm 9$ & $92 \pm 21$ & $56 \pm 25$ & $43 \pm 17$ \\
$50 \%$ & $17 \pm 9^{*}$ & $93 \pm 22$ & $56 \pm 27$ & $42 \pm 18$ \\
$75 \%$ & $19 \pm 10^{*}$ & $95 \pm 29$ & $56 \pm 27$ & $44 \pm 17$
\end{tabular}

${ }^{*} P<0.016$ with paired $t$ test with Bonferroni adjustment (50\% vs. $0 \%$ and $75 \%$ vs. $0 \%$ ); $n=30$.

Data are mean \pm SD.

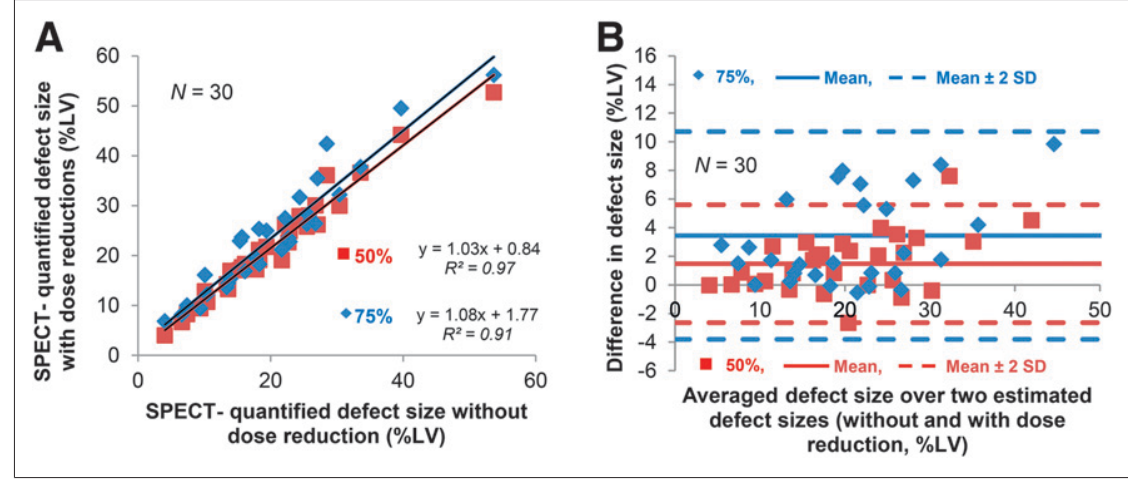

FIGURE 3. Analyses of myocardial perfusion defect size quantified from SPECT without AC: linear regression (A) and Bland-Altman (B) plots for comparisons of SPECT-quantified global defect sizes for $50 \%$ and $75 \%$ dose reductions vs. no dose reduction.
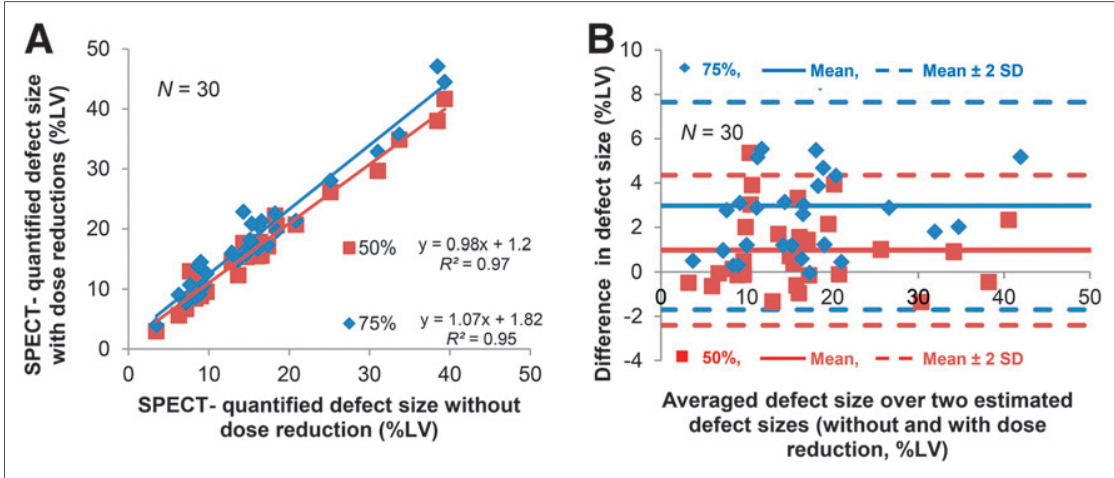

FIGURE 4. Analyses of myocardial perfusion defect size quantified from SPECT with AC: linear regression (A) and Bland-Altman (B) plots for comparisons of SPECT-quantified global defect sizes for $50 \%$ and $75 \%$ dose reductions vs. no dose reduction.

significant differences in the global and regional defect sizes resulting from the $75 \%$ dose reduction, and there was also a difference in the global defect size from the $50 \%$ reduction. However, these differences were miniscule, as demonstrated in Table 3, and might not be clinically significant.

\section{Effect of Dose Reduction on LV Function}

Overall comparisons of SPECT-quantified LV function among the $0 \%, 50 \%$, and $75 \%$ dose reductions for patients with normal and abnormal MPS results are shown in Tables 1 and 2, respectively. There were no significant differences in SPECTquantified EDV, ESV, or LVEF among the $0 \%, 50 \%$, and $75 \%$ dose reductions for patients without (Table 1) and with (Table 2) myocardial perfusion defects.

Figure 6 and Supplemental Figure 2, respectively, show the linear regression and Bland-Altman plots for SPECT-quantified $\mathrm{EDV}, \mathrm{ESV}$, and LVEF for quantitative comparisons of the original image $(0 \%$ reduction) versus the images with $50 \%$ and $75 \%$ dose reductions. As seen in Figure 6 and Table 4, the correlations were excellent and the differences were not statistically significant in either the overall comparison ( $n=30$, ANOVA) or the paired comparison ( $n=30$, paired $t$ with Bonferroni adjustment) of EDV, ESV, and LVEF. As demonstrated, the dose reductions simulated in this study did not have a manifest impact on the EDV, ESV, or LVEF quantifications in either patients without (Table 1) or patients with (Table 2) myocardial perfusion defects.

\section{DISCUSSION}

We have demonstrated that the exposure of patients to radiation can be reduced by at least $50 \%$ without compromising image quality or the quantitative precision of calculations of integrated myocardial perfusion defect size and global EDV, ESV, and LVEF. On the basis of the ANOVA statistics, the overall differences in global and regional perfusion defect sizes among the 3 groups $(0 \%, 50 \%$, and $75 \%$ dose reductions) were not statistically significant. For SPECT-quantified EDV, ESV, and LVEF, we also demonstrated in Tables 1, 2, and 4 that there was no significant difference in any comparison (3 overall groups; paired $50 \%$ vs. $0 \%$ and $75 \%$ vs. $0 \%$ dose reductions). Thus, the quantitative precision of the LV function assessment was not affected even when the dose was reduced by $75 \%$.

In this study, patients receiving the low dose were injected with an average of $360 \mathrm{MBq}(10 \mathrm{mCi})$ of ${ }^{99 \mathrm{~m}} \mathrm{Tc}$-tetrofosmin and images were acquired for $6 \mathrm{~min}$, whereas those receiving the high dose were injected with an average of $725 \mathrm{MBq}(20 \mathrm{mCi})$ and images were acquired for $4 \mathrm{~min}$. With the same two acquisition times (6 min for the high dose and 4 min for low dose), we expect that the dose can 

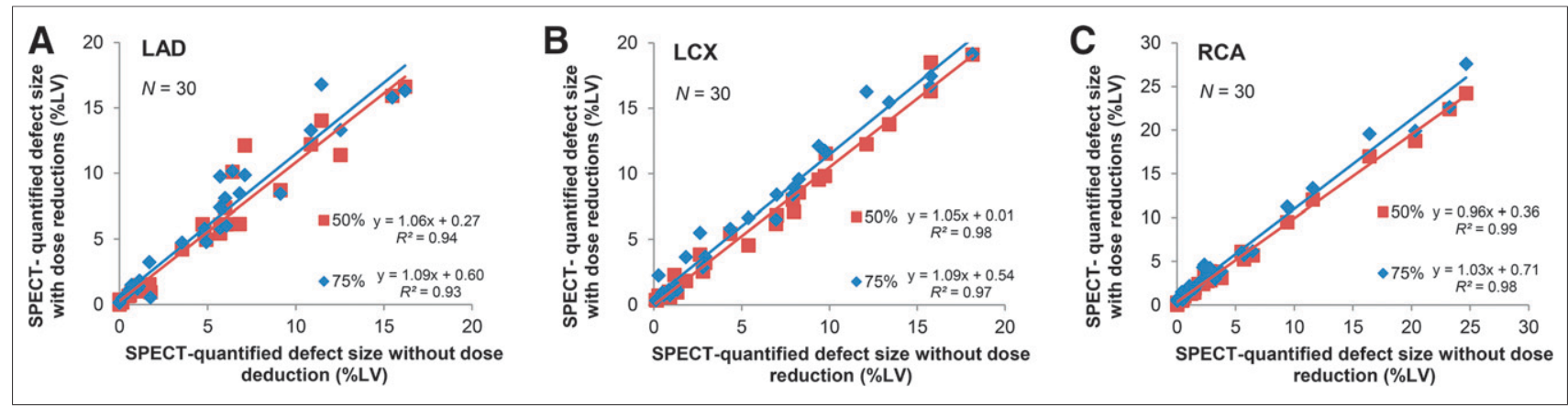

FIGURE 5. Linear regression analyses of agreement for comparisons of SPECT-quantified defect sizes for $50 \%$ and $75 \%$ dose reductions vs. no dose reduction, in left anterior descending $(A)$, left circumflex $(B)$, and right $(C)$ coronary artery territories.

be reduced by at least $50 \%$ to $180 \mathrm{MBq}(5 \mathrm{mCi})$ for the low dose and $363 \mathrm{MBq}(10 \mathrm{mCi})$ for the high dose. In a more aggressive manner, one can reduce the dose even further ( $75 \%$ dose reduction) to $90 \mathrm{MBq}(2.5 \mathrm{mCi})$ for the low dose and $180 \mathrm{MBq}(5 \mathrm{mCi})$ for the high dose if image quality is not noticeably compromised.

The current guidelines of the American Society of Nuclear Cardiology suggest ${ }^{99 m}$ Tc-tetrofosmin doses of 296-444 MBq (8-12 $\mathrm{mCi}$ ) for the low dose and 888-1,332 MBq (24-36 mCi) for the high dose in 1-d protocols. On the basis of the standard low dose of $370 \mathrm{MBq}(10 \mathrm{mCi})$ and high dose of $666 \mathrm{MBq}(18 \mathrm{mCi})$ currently used in the Yale-New Haven Hospital nuclear cardiac imaging laboratories, a new low dose of $185 \mathrm{MBq}(5 \mathrm{mCi})$ and high dose of 333 $\mathrm{MBq}(9 \mathrm{mCi})$ are considered appropriate when a similar CZT SPECT/ CT camera is used-a reduction of up to $75 \%$ from American Society of Nuclear Cardiology guidelines for the high dose and 58\% for the low dose. We believe that the dose can be potentially reduced by $75 \%$ without compromising the clinical diagnosis, attributable to the fact that the differences between SPECT-quantified defect sizes with and without the dose reductions were minimal even in the case of the $75 \%$ dose reduction.

As expected from the present study, the variability expressed as the SD of SPECT-quantified LVEF was larger for patients with abnormal MPS results (Table 2) than for those with normal results (Table 1), mainly because the abnormal cases included in this study had a wide LVEF range of $15 \%-71 \%$. Also, this EF variability was in part attributable to the relatively low count statistics of the 16-bin gated SPECT images that we regularly acquire in our laboratories. Nevertheless, on average, the EFs were nearly identical, ranging from $70 \%$ to $71 \%$ for normal-MPS patients (Table 1) and from $42 \%$ to $44 \%$ for abnormal-MPS patients (Table 2) among the 0\%, 50\%, and 75\% dose reductions. As evidenced by the fact that $\mathrm{EF}$ variability in normal-MPS patients (SD, $4 \%$ and 5\%; Table 1) was 3 times smaller than that in abnormal-MPS patients (SD, 17\% and 18\%; Table 2), the large EF variation, particularly in the abnormal group, was in fact caused by the wide range of EF and low count statistics of 16-bin electrocardiogram-gated SPECT attributable to myocardial perfusion defects. Apparently, the dose reduction itself did not contribute to the EF variability, as demonstrated in Tables 1 and 2 .

Previous studies on dose reductions from half-time or quarter-time imaging were mostly derived from and supported by the wide-beam SPECT reconstruction (26-28) in which conventional rotating cameras are used and images are reconstructed without CT-based AC. In the present study, however, we used a hybrid SPECT/CT system with stationary CZT SPECT detectors, and the images were reconstructed via the iterative maximum-likelihood expectation maximization algorithm with CT-based AC. A more recent study on dose reduction using IQ-SPECT was reported by Caobelli et al. (29), who claimed that one-eighth-time gated MPS was feasible. However, they used conventional 8-bin electrocardiogram-gated SPECT, which had higher count statistics than the 16-bin electrocardiogram-gated SPECT that we used in the present study. Also, their study did not include a population of normal-perfusion subjects but only patients with suspected or diagnosed coronary artery disease. Although the SPECT count distribution in the myocardium of patients with normal myocardial perfusion (Fig. 1) is usually homogeneous, an excessive dose reduction may cause heterogeneity because of suboptimal count statistics, resulting in a false-positive diagnosis of abnormal myocardial perfusion. We demonstrated in this report that dose reductions in the 10 low-risk patients did not generate false-positive perfusion defects, regardless of whether a $50 \%$ or $75 \%$ dose reduction was used (Table 1). Furthermore, the EDV, ESV, and LVEF derived from $50 \%$ and $75 \%$ dose reductions were exceedingly consistent with those derived without dose reduction, as shown in Table 1. Nevertheless, a false-positive result might still be possible if the image quality is severely compromised by an exceptionally aggressive dose reduction, such as the one-eighthtime gated SPECT used by Caobelli et al. (29).

Although similar dose reduction studies for SPECT without AC have been performed by others $(14-16,20,22)$, the present study

TABLE 3

Changes in Regional and Global Myocardial Perfusion

Defect Sizes for $50 \%$ and $75 \%$ Dose Reductions vs. No

Dose Reduction, in Patients with Myocardial Perfusion Defects

\begin{tabular}{|c|c|c|c|c|}
\hline \multirow[b]{2}{*}{$\begin{array}{l}\text { Dose } \\
\text { reduction }\end{array}$} & \multicolumn{4}{|c|}{ Change in defect size in... } \\
\hline & $\begin{array}{l}\text { Left anterior } \\
\text { descending } \\
\text { coronary } \\
\text { artery (\%LV) }\end{array}$ & $\begin{array}{c}\text { Left } \\
\text { circumflex } \\
\text { coronary } \\
\text { artery } \\
\text { (\%LV) }\end{array}$ & $\begin{array}{l}\text { Right } \\
\text { coronary } \\
\text { artery } \\
\text { (\%LV) }\end{array}$ & $\begin{array}{c}\text { Entire } \\
\text { left } \\
\text { ventricle } \\
(\% \text { LV) }\end{array}$ \\
\hline $50 \%$ & $0.55 \pm 1.28$ & $0.28 \pm 0.75$ & $0.13 \pm 0.58$ & $0.97 \pm 1.69^{*}$ \\
\hline $75 \%$ & $1.05 \pm 1.44^{*}$ & $1.06 \pm 1.06^{\star}$ & $0.85 \pm 0.94^{\star}$ & $2.96 \pm 2.35^{\star}$ \\
\hline \multicolumn{5}{|c|}{$\begin{array}{l}{ }^{\star} P<0.016 \text { with paired } t \text { test with Bonferroni adjustment; } \\
=30 . \\
\text { Data are mean } \pm \text { SD. }\end{array}$} \\
\hline
\end{tabular}



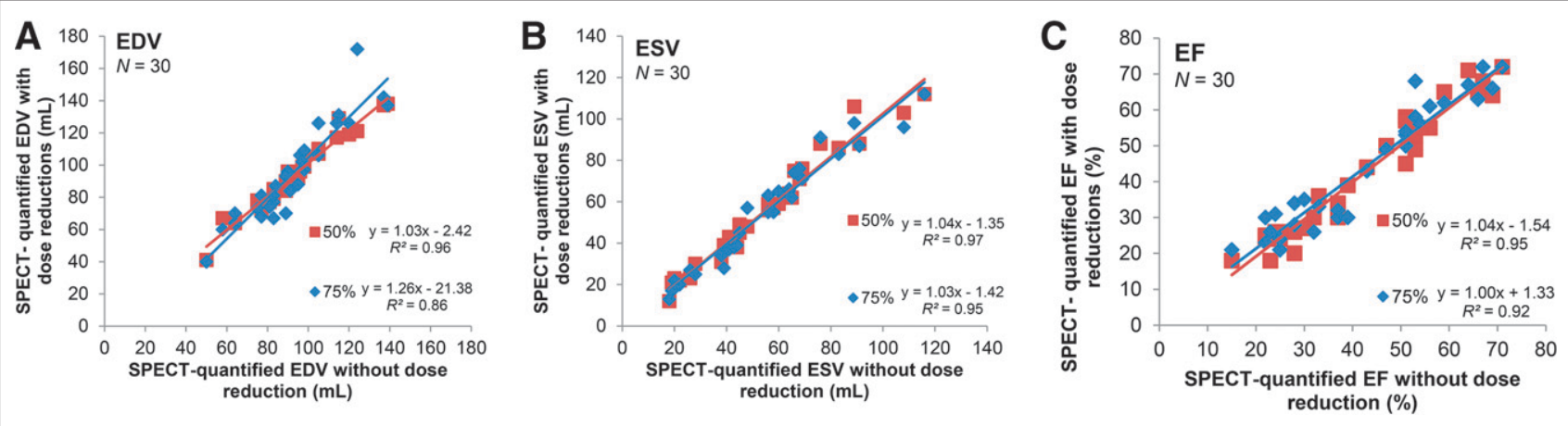

FIGURE 6. Linear regression analyses of agreement for comparisons of SPECT-quantified EDV (A), ESV (B), and EF (C) for $50 \%$ and $75 \%$ dose reductions vs. no dose reduction.

TABLE 4

Changes in EDV, ESV, and EF in Patients with Myocardial Perfusion Defects

\begin{tabular}{lcrr}
\hline \multirow{2}{*}{ Dose reduction } & \multicolumn{3}{c}{ Change in... } \\
\cline { 2 - 4 } & EDV $(\mathrm{mL})$ & ESV $(\mathrm{mL})$ & \multicolumn{1}{c}{ EF $(\%)$} \\
\hline $50 \%$ & $0.73 \pm 4.48$ & $0.8 \pm 5.30$ & $-0.03 \pm 3.99$ \\
$75 \%$ & $2.57 \pm 12.31$ & $0.03 \pm 5.99$ & $1.43 \pm 5.00$
\end{tabular}

$P=$ not statistically significant with paired $t$ test with Bonferroni adjustment; $n=30$.

Data are mean \pm SD.

focused mainly on the differential effect of dose reduction on quantification from SPECT with AC. Additionally, we also analyzed the sizes of myocardial perfusion defects from SPECT without AC, and the results were comparable to those from SPECT with AC, implying that the use of AC did not affect reproducibility or differences in perfusion defects between the high and low doses.

The hybrid SPECT/CT camera we used may not be widely available in nuclear cardiac imaging laboratories. Thus, our findings on the dose reductions may not be immediately adaptable to sites without this type of high-end imaging system. Nevertheless, one can still reduce the injected dose while increasing the acquisition time to preserve the desired count statistics. Radiation safety has become a major concern in nuclear cardiology, and to maintain good image quality and precise quantitative results in daily routine practice, one may prefer to prolong the acquisition while reducing the dose.

\section{CONCLUSION}

A $50 \%$ reduction of the already-reduced clinical doses using a CZT SPECT/CT camera is highly feasible and may not markedly affect image quality or SPECT quantification. A reduction of ${ }^{99 \mathrm{~m}} \mathrm{Tc}-$ labeled myocardial perfusion tracers to $185 \mathrm{MBq}(5 \mathrm{mCi})$ for the low dose and $333 \mathrm{MBq}(9 \mathrm{mCi})$ for the high dose using CZT SPECT/CT is exceedingly appropriate even with short acquisitions.

\section{DISCLOSURE}

The costs of publication of this article were defrayed in part by the payment of page charges. Therefore, and solely to indicate this fact, this article is hereby marked "advertisement" in accordance with 18 USC section 1734. No potential conflict of interest relevant to this article was reported.

\section{ACKNOWLEDGMENT}

This study was presented in part at the International Conference on Nuclear Cardiology and Cardiac CT (ICNC 12), May 2015, Madrid, Spain.

\section{REFERENCES}

1. DePuey EG, Nichols K, Dobrinsky C. Left ventricular ejection fraction assessed from gated technetium-99m-sestamibi SPECT. J Nucl Med. 1993;34:1871-1876.

2. Germano G, Kiat H, Kavanagh PB, et al. Automatic quantification of ejection fraction from gated myocardial perfusion SPECT. J Nucl Med. 1995;36:2138-2147.

3. Liu YH, Sinusas AJ, DeMan P, Zaret BL, Wackers FJT. Quantification of SPECT myocardial perfusion images: methodology and validation of the Yale-CQ method. J Nucl Cardiol. 1999;6:190-204.

4. Shen MYH, Liu YH, Sinusas AJ, et al. Quantification of regional myocardial wall thickening on electrocardiogram-gated SPECT imaging. J Nucl Cardiol. 1999;6:583-595.

5. Navare SM, Wackers FJ, Liu YH. Comparison of 16-frame and 8-frame gated SPET imaging for determination of left ventricular volumes and ejection fraction. Eur J Nucl Med Mol Imaging. 2003;30:1330-1337.

6. Liu YH, Sinusas AJ, Khaimov D, Gebuza BI, Wackers FJ. New hybrid countand geometry-based method for quantification of left ventricular volumes and ejection fraction from ECG-gated SPECT: methodology and validation. $\mathrm{J}$ Nucl Cardiol. 2005;12:55-65.

7. Wackers FJ, Bruni W, Zaret BL, eds. Nuclear Cardiology: The Basics. 2nd ed. Totowa, New Jersey: Humana Press Inc; 2008.

8. Liu YH, Wackers FJ, eds. Cardiovascular Imaging. London, U.K.: Manson Publishing, Ltd.; 2009.

9. Slomka PJ, Patton JA, Berman DS, Germano G. Advances in technical aspects of myocardial perfusion SPECT imaging. J Nucl Cardiol. 2009;16:255-276.

10. LaCroix K, Tsui B, Hasegawa B, Brown J. Investigation of the use of X-ray CT images for attenuation compensation in SPECT. IEEE Trans Nucl Sci. 1994;41:2793-2799.

11. Masood Y, Liu YH, Depuey G, et al. Clinical validation of SPECT attenuation correction using $\mathrm{X}$-ray computed tomography-derived attenuation maps: multicenter clinical trial with angiographic correlation. J Nucl Cardiol. 2005;12:676-686.

12. Tsui B, Hu H, Gilland D, Gullberg G. Implementation of simultaneous attenuation and detector response correction in SPECT. IEEE Trans Nucl Sci. 1988; NS-35:778-783.

13. Henzlova MJ, Duvall WL. The future of SPECT MPI: time and dose reduction. J Nucl Cardiol. 2011;18:580-587.

14. Duvall WL, Croft LB, Godiwala T, Ginsberg E, George T, Henzlova MJ. Reduced isotope dose with rapid SPECT MPI imaging: initial experience with a CZT SPECT camera. J Nucl Cardiol. 2010;17:1009-1014.

15. Herzog BA, Buechel RR, Katz R, et al. Nuclear myocardial perfusion imaging with a cadmium-zinc-telluride detector technique: optimized protocol for scan time reduction. J Nucl Med. 2010;51:46-51. 
16. Duvall WL, Croft LB, Ginsberg ES, et al. Reduced isotope dose and imaging time with a high-efficiency CZT SPECT camera. J Nucl Cardiol. 2011;18:847857.

17. Nkoulou R, Pazhenkottil AP, Kuest SM, et al. Semiconductor detectors allow low-dose-low-dose 1-day SPECT myocardial perfusion imaging. J Nucl Med. 2011;52:1204-1209.

18. Duvall WL, Sweeny JM, Croft LB, Ginsberg E, Guma KA, Henzlova MJ. Reduced stress dose with rapid acquisition CZT SPECT MPI in a non-obese clinical population: comparison to coronary angiography. J Nucl Cardiol. 2012;19:19-27.

19. Gimelli A, Bottai M, Genovesi D, Giorgetti A, Di Martino F, Marzullo P. High diagnostic accuracy of low-dose gated-SPECT with solid-state ultrafast detectors: preliminary clinical results. Eur J Nucl Med Mol Imaging. 2012;39:83-90.

20. Nakazato R, Berman DS, Hayes SW, et al. Myocardial perfusion imaging with a solid-state camera: simulation of a very low dose imaging protocol. J Nucl Med. 2013;54:373-379.

21. Garcia EV, Faber TL, Esteves FP. Cardiac dedicated ultrafast SPECT cameras: new designs and clinical implications. J Nucl Med. 2011;52:210-217.

22. Esteves FP, Raggi P, Folks RD, et al. Novel solid-state-detector dedicated cardiac camera for fast myocardial perfusion imaging: multicenter comparison with standard dual detector cameras. J Nucl Cardiol. 2009;16:927-934.
23. Buechel RR, Herzog BA, Husmann L, et al. Ultrafast nuclear myocardial perfusion imaging on a new gamma camera with semiconductor detector technique: first clinical validation. Eur J Nucl Med Mol Imaging. 2010;37:773-778.

24. Liu YH. Quantification of nuclear cardiac images: the Yale approach. $J$ Nucl Cardiol. 2007;14:483-491.

25. Bland JM, Altman DG. Statistical methods for assessing agreement between two methods of clinical measurement. Lancet. 1986;1:307-310.

26. DePuey EG, Bommireddipalli S, Clark J, Thompson L, Srour Y. Wide beam reconstruction "quarter-time" gated myocardial perfusion SPECT functional imaging: a comparison to "full-time" ordered subset expectation maximum. J Nucl Cardiol. 2009; 16:736-752.

27. Druz RS, Phillips LM, Chugkowski M, Boutis L, Rutkin B, Katz S. Wide-beam reconstruction half-time SPECT improves diagnostic certainty and preserves normalcy and accuracy: a quantitative perfusion analysis. J Nucl Cardiol. 2011;18:52-61.

28. Marcassa C, Campini R, Zoccarato O, Calza P. Wide beam reconstruction for half-dose or half-time cardiac gated SPECT acquisitions: optimization of resources and reduction in radiation exposure. Eur J Nucl Med Mol Imaging. 2011;38:499-508.

29. Caobelli F, Thackeray JT, Soffientini A, Bengel FM, Pizzocaro C, Guerra UP. Feasibility of one-eighth time gated myocardial perfusion SPECT functional imaging using IQ-SPECT. Eur J Nucl Med Mol Imaging. 2015;42:1920-1928. 